\title{
on the random distribution of numbers
}

There was a time, not long ago, when physicists and mathematicians (even though they really knew better) sought a way to generate finite series of random numbers. I don't recall all the details, but in the end., most concluded that by some program or whatever, computers would be effective random number generators.

At this juncture, you may be wondering, as I am, why bother? What is the sense in generating a series of numbers that are truly random. Truth be told, I really don't know. It seems that randomness is needed in order for us to tell time with precision, or to exactly determine what is not random, which I think is the major endpoint. But to a scientist like me, the very impossibility of generating random numbers, a limited numbers of numbers that randomly differ (of course, if the set were unlimited, then its randomness would be rather moot, as an infinite number of each number would derive, and would so regardless of the relative magnitude of any of the numbers. That means that in an infinite set, assuming infinity / infinity $=1$, if there are 10 times as many number $10 \mathrm{~s}$ as ones, there shall derive, when the counting is done (a day that can never come) as many of both (go figure).

It is, therefore, intuitively clear that nothing is infinite, unless one considers the facts not known, and therein lies one problem; no one knows. Figuring correctly that density $d=m / v$, Big Al calculated the estimated size of our finite universe; an infinite universe would lead to no mass anywhere, as $\mathrm{m} / \mathrm{v}$ would be zero. Which gets us somewhat closer to the problem; what is zero? What is nothing? Of this, we have no concept, but it certainly is not empty space. What is nothing; it is nothing, a concept we cannot understand at all, but one that must exist. (must?).

[In its final alalysis, the basic argumemts are not dissimilar than those of relativity. $c$ is the limiting velocity of all things. Absolute zero is the temperature we expect to result from (not in) cessation of all movement (yes, subatomic). This temperature cannot be reached. We fail to consider a maximal $\mathrm{T}$, which is the highest achievable temperature and we do so because we think that it can always get hotter (like infinity, we can always travel farther from our starting point, right? Wrong).

Well, when the atoms move at the speed of light, then it can't get no hotter so there is an absolute heat. Pushing the rapidly moving atoms together would make it hotter, but the heat generated would put a finite limit on how close they can get, so there is an absolute hot. Probably very close to the heat within the so called big bang. And similarly, unless a distance of no distance can be achieved (it cannot), then a distance of infinity cannot either (in contrast, in an infinite universe, absolute zero would be theoretically possible and mass could travel at $\mathrm{v}=\mathrm{c}$ or higher).

Back to randomness, one may argue that nothing is in a finite universe, and one can argue that everything is. In fact, this basic difference in ideology was the basis of the rift between Professor Einstein and Neils Bohr, culminating in Einstein's assertion that "God does not play dice with the universe" and Professor Bohr's adroit response, "My Dear Dr. Einstein, I do not believe that God plays dice".

Are both right? Perhaps. Thus, Einstein could not envision that any particle did not hold an exact position even if that position was not determinable. Quanta, which no one really understands, holds that all do; the smaller, the less precise. Not only is the position (and velocity) of all things undeterminable, quanta holds that both (and either) entity does not in fact exist. What does exist is a fairly certain probability of the objects position and velocity at any specific time (fairly certain?)

There are many ramifications to the two opposing views. If $\mathrm{Al}$ is right, everything is certain; if all the facts are known, and they could be were it not for that nagging principle of uncertainty of Hertenzenberg, then we could determine our fate; further, our fate is certainly already determined, and will power or volition, forget about it.

If Bohr is correct, all bets are off and what will be will depend on chance.

I don't intend to argue the two here, but let's assume quanta is correct if only because most do.
If quanta truly represent uncertainty, then it is possible to generate randomness. The closest we have come is the randomness of atomic nuclei decay. Now this is one difficult thing to deal with. Here, we have a population of nuclei surrounded by electrons that decay at a very predictable rate. However, the decay of any one member of this population is impossible to determine and in fact its probability is exactly the same as that of the decay of all other members of the population. Regardless of the individual history (age) of the atom in question. For example, half of the atoms of radioactive phosphate, P-32, decay by internal conversion of a neutron to a proton and Azuer electron in something like 6 days (the half life is exactly known). Regardless of the environment, temperature, and importantly age of the atom. All of the atoms are thought to be identical, regardless of their age. A P-32 atom that has survived decay for a month (a possibility of about $1 / 2^{4}$ or $1 / 16$ ) has the same possibility of decaying in the next moment as one that is recently born. This surprising fact runs counter to the half life of all other things. Humans may have a half life of 40 years, but 40 year old humans have a half life of much less. So do stars, rocks, and everything known. But everything known is composed only of atoms with a determined and unchanging half life, atoms that do not, in essence, age. So why does everything else age in a manner that differs from that of individual atoms. This reflects the fact ${ }^{8}$ basis of existence of all things in the universe we know to exist.

Thus, while atomic decay represents perhaps the most random event known, it is also the most precise. Nuclear clocks depend on measurement of the rate of atomic decay, and no clock is and just as it is certain that the future shall arrive, it is certain that the future shall arrive randomly.

Truly, random numbers are within any range must similarly exhibit a certain and precise deviation.

Suppose that range is $0-100$ such that the mean is 50 . One could experimentally determine the range of randomness in this case by using a radioactive plasma with a half life of 50 ( ok, per minute). A large number of atoms in this popula- 
tion would survive and an equally large number would decay within one minute. The question on the table is how many? This cannot be determined, but the more atoms assessed, the closer the number will be to $1 / 2$ of the initial number. If $10^{8}$ atoms are initially present, the $0.5 \times 10^{8}$ $+/$ - some number that is known and that represents perhaps less that ' $1 \times 10^{-3}$ of the number decayed (or less that .1\%) would differ from the mean in a manner we term "significant". If however, if only 100 atoms are initially present, then the variation would be markedly higher. While the mean may be close to 50 , fully $15 \%$ of the atoms would defy the expected decay within that minute. One standard deviation from whatever mean is then determined as amount that accounts for $66 \%$ of the observations. This would differ from the mean by at least $15 \%$ (this again is the nature of randomness only and is unavoidable).

Since the purpose of discovery is to determine what is not known, the half life of a population of atoms with a true half life of 50 would be accurate (within one SD of the real half life) $66 \%$ of the time and would thereby vary from a number of about 35-65 with confidence if 100 observations were made (or if 100 atoms) were observed. If a few billion were observed, the portion of the range of deviation from the mean would be much less. Determination of the difference in the half life of any other population from the one in question, which was unknown at the initiation of the experiment, would be dependent upon the number of atoms studied only. A population of atoms with a true half life between 35 and 65 would be considered to have NO DIFFERENT half life than that of the population with a half life of 50, if 100 atoms were studied. If a billion were, differences in half life of less that $1 \%$ would readily be evident. Thus in analyses of patient populations in a clinical trial of 100 patients in each of 2 arms, NO DIFFERENCE would be confidently determined in an individual study even if the real difference was over $15 \%$ of the patients studied. Further, with such a low sample size, it can be predicted that 100 studies would show a difference with confidence over $30 \%$ of the time $(15 \%$ would show a beneficial effect and 15\% would show a detrimental effect.

doi : 10.5214/ans.0972.7531.1118401

\section{Denis English, PhD}

Senior Editor 